\title{
The importance of distinguishing pseudogenes from parental genes
}

\author{
Luke B Hesson ${ }^{*}$ and Robyn L Ward
}

\section{Dear Editor,}

The July-August 2014 issue of Clinical Epigenetics featured a research article describing PTEN promoter hypermethylation in multiple myeloma by Piras et al. [1]. The importance of the PTEN gene has resulted in significant efforts to identify sequence, expression and methylation changes in cancer. Piras et al. concluded that PTEN hypermethylation occurred in a subset of multiple myeloma cases but that hypermethylation did not correlate with reduced gene expression or clinical parameters. The PTEN mRNA shares $97.8 \%$ sequence identity with a pseudogene known as PTENP1. A 921-bp region of the promoters of these genes is also $91 \%$ identical. Consequently, careful consideration of assay design is required to avoid amplification of PTENP1 rather than PTEN sequences. However, the method used by Piras et al. for measuring PTEN mRNA did not distinguish between these homologues, despite numerous studies showing that PTENP1 mRNA is ubiquitously expressed in both normal and cancer specimens [2-5]. Furthermore, previous studies have demonstrated that apparent methylation of the PTEN promoter is likely attributable to the non-specific amplification of the highly homologous PTENP1 gene $[6,7]$. We have shown that the only reliable method for distinguishing between PTEN and PTENP1 promoter methylation is single-molecule bisulfite sequencing that utilizes sequence differences between the two genes to separately analyze individual promoter molecules $[6,8]$. These methodological challenges make comparisons between methylation and expression impossible when using assays that do not reliably discriminate between PTEN and PTENP1, and also negate the value of correlating these features with clinicopathological characteristics.

The challenges posed by sequence homology with pseudogenes are by no means particular to the PTEN gene. For example, the DNA mismatch repair gene PMS2 shares $>95.2 \%$ sequence identity with at least six other genes (PMS2CL, PMS2L2, PMS2P4, PMS2P5, PMS2P1 and PMS2P11) making analysis of the PMS2 CpG island promoter region particularly challenging.

In light of the recent manuscript by Piras et al., it is necessary to highlight the importance of rigorous methodology when investigating DNA methylation changes in cancer, especially concerning genes with homologues or pseudogenes such as PTEN.

\section{Competing interests}

The authors declare that they have no competing interests.

Received: 28 November 2014 Accepted: 28 November 2014 Published online: 31 December 2014

\section{References}

1. Piras G, Monne M, Palmes a, Calvisi A, Asproni R, Vacca F, Pilo L, Gabbas A, Latte G: Methylation analysis of the phosphates and tensin homologue on chromosome 10 gene (PTEN) in multiple myeloma. Clin Epigenetics 2014, 6:16.

2. Fujii GH, Morimoto AM, Berson AE, Bolen JB: Transcriptional analysis of the PTEN/MMAC1 pseudogene, psiPTEN. Oncogene 1999, 18:1765 1769.

3. loffe YJ, Chiappinelli KB, Mutch DG, Zighelboim I, Goodfellow PJ: Phosphatase and tensin homolog (PTEN) pseudogene expression in endometrial cancer: a conserved regulatory mechanism important in tumorigenesis? Gynecol Oncol 2012, 124:340 346.

4. Yokoyama Y, Wan X, Shinohara A, Takahashi S, Takahashi Y, Niwa K, Tamaya $\mathrm{T}$ : Expression of PTEN and PTEN pseudogene in endometrial carcinoma. Int J Mol Med 2000, 6:47 50.

5. Zhang CL, Tada M, Kobayashi H, Nozaki M, Moriuchi T, Abe H: Detection of PTEN nonsense mutation and psiPTEN expression in central nervous system high-grade astrocytic tumors by a yeast-based stop codon assay. Oncogene 2000, 19:4346 4353.

6. Hesson LB, Packham D, Pontzer E, Funchain P, Eng C, Ward RL: A reinvestigation of somatic hypermethylation at the PTEN CPG island in cancer cell lines. Biol Proced Online 2012, 14:5.

7. Zysman MA, Chapman WB, Bapat B: Considerations when analyzing the methylation status of PTEN tumor suppressor gene. Am J Pathol 2002, 160:795 800 .

8. Hesson LB, Ward RL: Discrimination of pseudogene and parental gene DNA methylation using allelic bisulfite sequencing. Methods Mol Biol 2014, 1167:265 274.

doi:10.1186/s13148-014-0033-5

Cite this article as: Hesson and Ward: The importance of distinguishing pseudogenes from parental genes. Clinical Epigenetics 2014 6:33.

* Correspondence: I.hesson@unsw.edu.au

Adult Cancer Program, Lowy Cancer Research Centre and Prince of Wales

Clinical School, UNSW, Sydney, Australia 